\title{
Social isolation and loneliness as risk factors for myocardial infarction, stroke and mortality: UK Biobank cohort study of 479054 men and women
}

\author{
Christian Hakulinen, 1,2 Laura Pulkki-Råback, ${ }^{1}$ Marianna Virtanen, ${ }^{3,4}$ Markus Jokela, ${ }^{1}$ \\ Mika Kivimäki, ${ }^{5,6}$ Marko Elovainio ${ }^{1,2}$
}

- Additional material is published online only. To view please visit the journal online (http://dx.doi.org/10.1136/ heartjnl-2017-312663).

'Department of Psychology and Logopedics, Faculty of Medicine, University of Helsinki, Helsinki, Finland

${ }^{2}$ National Institute for Health and Welfare, Helsinki, Finland ${ }^{3}$ Finnish Institute of

Occupational Health, Helsinki, Finland

${ }^{4}$ Department of Public Health and Caring Sciences, University of Uppsala, Uppsala, Sweden ${ }^{5}$ Department of Epidemiology and Public Health, University College London, London, UK ${ }^{6}$ Clinicum, Faculty of Medicine, University of Helsinki, Helsinki, Finland

\section{Correspondence to}

Dr Christian Hakulinen, Department of Psychology and Logopedics, Faculty of Medicine, University of Helsinki, Helsinki, Finland; christian.hakulinen@ helsinki.fi

Received 1 November 2017 Revised 20 February 2018 Accepted 25 February 2018 Published Online First 27 March 2018
Check for updates

To cite: Hakulinen C, PulkkiRåback L, Virtanen M, et al. Heart 2018;104:1536-1542.

\begin{abstract}
Objective To examine whether social isolation and loneliness (1) predict acute myocardial infarction (AMI) and stroke among those with no history of AMI or stroke, (2) are related to mortality risk among those with a history of AMI or stroke, and (3) the extent to which these associations are explained by known risk factors or pre-existing chronic conditions.

Methods Participants were 479054 individuals from the UK Biobank. The exposures were self-reported social isolation and loneliness. AMI, stroke and mortality were the outcomes.
\end{abstract}

Results Over 7.1 years, 5731 had first AMI, and 3471 had first stroke. In model adjusted for demographics, social isolation was associated with higher risk of AMI (HR 1.43, 95\% Cl 1.3 to -1.55) and stroke (HR 1.39, $95 \% \mathrm{Cl} 1.25$ to 1.54$)$. When adjusted for all the other risk factors, the HR for AMI was attenuated by $84 \%$ to 1.07 (95\% $\mathrm{Cl} 0.99$ to 1.16$)$ and the HR for stroke was attenuated by $83 \%$ to $1.06(95 \% \mathrm{Cl} 0.96$ to 1.19$)$. Loneliness was associated with higher risk of AMI before (HR 1.49, $95 \% \mathrm{Cl} 1.36$ to 1.64) but attenuated considerably with adjustments (HR $1.06,95 \% \mathrm{Cl} 0.96$ to 1.17). This was also the case for stroke (HR $1.36,95 \% \mathrm{Cl}$ 1.20 to 1.55 before and HR $1.04,95 \% \mathrm{Cl} 0.91$ to 1.19 after adjustments). Social isolation, but not loneliness, was associated with increased mortality in participants with a history of AMI (HR $1.25,95 \% \mathrm{Cl} 1.03$ to 1.51) or stroke (HR $1.32,95 \% \mathrm{Cl} 1.08$ to 1.61 ) in the fully adjusted model.

Conclusions Isolated and lonely persons are at increased risk of AMI and stroke, and, among those with a history of AMI or stroke, increased risk of death. Most of this risk was explained by conventional risk factors.

\section{INTRODUCTION}

Individuals who are socially isolated (ie, are lacking social contacts and participation in social activities) or feel lonely (ie, feel that they have too few social contacts or are not satisfied with the quality of their social contacts) have been found to be at increased risk of incident coronary heart disease (CHD), ${ }^{1}$ stroke $^{2}$ and early mortality. ${ }^{3-7}$ A recent meta-analysis-including 11 longitudinal studies on cardiovascular disease and 8 on stroke-suggested that social isolation and loneliness are associated with 30\% excess risk of incident CHD and stroke. ${ }^{8}$ However, most of the studies were small in scale, with only one study reporting more than
1000 events, ${ }^{1}$ and meta-analytic evidence suggests selective publishing of positive findings. ${ }^{8}$ Furthermore, only a limited set of potential explanatory factors have been examined in previous studies and mortality after incident CHD or stroke remains unexplored. Thus, it remains unclear whether these associations are independent of biological, behavioural, psychological, health and socioeconomic factors ${ }^{9-11}$ that are known to increase risk of cardiovascular diseases. ${ }^{12} 13$ In addition, although other risk factors, such as physical inactivity ${ }^{14}$ and depression, ${ }^{15}$ have been associated with poorer outcomes among individuals with pre-existing cardiovascular disease, it remains unclear whether socially isolated or lonely individuals have an elevated risk of early mortality after cardiovascular disease event.

In this analysis using the UK Biobank study, a very large prospective population-based cohort study, we examined the associations of social isolation and loneliness with first acute myocardial infarction (AMI) and first stroke. In addition, we examined whether social isolation and loneliness before AMI or stroke event are associated with mortality risk after the event. A broad range of biological, behavioural, psychological, socioeconomic and mental health-related factors were included as potential mediators or confounders of these associations.

\section{METHODS}

\section{Study design}

In total, 502632 participants (aged 40-69 years) were recruited to the UK Biobank study between April 2007 and December 2010 from the general population $(5.5 \%$ response rate). Participants completed touch-screen questionnaire, had physical measurements taken and biological samples collected by trained data nurses in one of the 22 assessment centres across England, Wales and Scotland. Details of these have been reported elsewhere. ${ }^{16} 17$ In the current study, social isolation and loneliness were used as exposures and AMI, stroke and mortality after AMI or stroke events as outcomes. The present study sample was restricted to the 479054 participants who had complete data on either social isolation or loneliness, and AMI and stroke. A total of 18704 participants were excluded due to history of AMI or stroke before the baseline. 


\section{Procedures}

Date of death was obtained from death certificates held by the National Health Service (NHS) Information Centre (England and Wales) and the NHS Central Register Scotland (Scotland). Hospital admissions were identified via record linkage to Hospital Admitted Patient Care Activity (England), General/ Acute Inpatient and Day Case dataset (Scotland), and Patient Episode Database for Wales. AMI and stroke events were recorded from the death register and hospital admission using the following International Classification of Diseases (ICD)-10 codes: AMI: I21.X, I22.X, I23.X, I24.1 and I25.2; stroke: I60, I61, I63 and I64.

Age was calculated based on birth month and year. Ethnicity was defined as Caucasian versus other based on self-reported ethnicity. Educational attainment was categorised into three groups (no secondary education, secondary education and university degree), and annual household income was measured with a five-point scale (less than $£ 31000, £ 18000$ to $£ 29999$, $£ 30000$ to $£ 51999, £ 52000$ to $£ 100000$ and greater than $£ 100$ 000). Area-based socioeconomic status was derived from postcode of residence using the Townsend Deprivation Index score. ${ }^{18}$

Social isolation and loneliness were assessed with scales that were used in a previous UK Biobank study. ${ }^{7}$ The social isolation scale contained three questions ((1) "Including yourself, how many people are living together in your household?"; (2) "How often do you visit friends or family or have them visit you?"; and (3) "Which of the following (leisure/social activities) do you engage in once a week or more often? You may select more than one"), where certain answers were given one point (1 point for no participation in social activities at least weekly; 1 point for living alone; 1 point for friends and family visits less than once a month), and all other answers 0 point. This resulted in a scale ranging from 0 to 3 where person was defined as socially isolated if she/he had two or more points. Loneliness was measured with two questions: "Do you often feel lonely?" (no=0, yes=1) and "How often are you able to confide in someone close to you?" $(0=$ almost daily to once every few months; $1=$ never or almost never). An individual was defined as lonely if she/he answered positively to both questions (score 2). Similar questions are used in other social isolation and loneliness scales (eg, Revised UCLA Loneliness Scale ${ }^{19}$ ).

Height and weight were measured at the clinic, and body mass index (BMI) was calculated as weight/height $(\mathrm{m})^{2}$. Grip strength was measured using Jamar (model J00105) hydraulic hand dynamometer and the mean of the right-hand and lefthand values was calculated and used in the analyses. Cigarette smoking (current smoker (yes/no); ex-smoker (yes/no)), physical activity (moderate and vigorous) and alcohol-intake frequency (three or four times a week or more vs once or twice a week or less) were self-reported. Depressive symptoms were assessed with the following four questions from the Patient Health Questionnaire ${ }^{20}$ : the frequency of (1) depressed mood, (2) disinterest or absence of enthusiasm, (3) tenseness or restlessness, and (4) tiredness or lethargy in the previous 2 weeks. Current chronic diseases (diabetes, cardiovascular disease, cancer and other longstanding illness, disability or infirmity) was categorised into yes versus no. Further details of these measures can be found in the UK Biobank online protocol (http://www.ukbiobank.ac.uk/).

\section{Statistical analyses}

Descriptive statistics are presented as mean (SD of the mean) or number (percentage) for continuous and categorical variables, respectively. Associations between social isolation and loneliness with incident AMI, stroke and mortality after AMI or stroke were examined using Cox proportional hazards models where age was used as the timescale, ${ }^{21}$ and birth month and year as time origin. The proportional hazards assumption was graphically investigated using $\log -\log$ plots and Schoenfeld residual plots, and no major violations were observed. AMI, stroke and mortality after AMI or stroke were examined as separate outcomes. Age, sex and ethnicity were used as covariates in all models. Subgroup analyses were conducted separately for men and women, three age groups (37-52 years; 53-60 years; 61-73 years) and ethnic groups (white vs non-white) as these can be seen as potential confounders.

To examine the extent to which baseline biological, behavioural, socioeconomic, psychological and health-related risk factors explained the associations, percentage of excess risk mediated (PERM) was calculated for the following mechanisms: (1) biological (BMI, diastolic and systolic blood pressure, grip strength); (2) behavioural (alcohol consumption, physical activity and smoking); (3) socioeconomic (education, household income and Townsend Deprivation Index) and (4) mental health (depressive symptoms); and (5) history of chronic illness. PERM was calculated using the following formula ${ }^{22}$ :

$$
\begin{aligned}
\text { PERM }= & {\left[\mathrm{HR}_{(\text {age, sex and ethnicity adjusted })}\right.} \\
& \left.-\mathrm{HR}_{(\text {age, sex, and ethnicity and risk factor adjusted })}\right] \\
& /\left[\mathrm{HR}_{(\text {age, sex and ethnicity adjusted })}-1\right] \times 100
\end{aligned}
$$

Missing data were imputed with multiple imputation procedure using the chained equations method. ${ }^{23}$ In total, five imputed datasets were generated and results were combined using Rubin's rules. Imputation model included basic demographics (age, sex and ethnicity), predictors (social isolation and loneliness), all mediating variables, the Nelson-Aalen estimate of cumulative hazard, and AMI and stroke status. All statistical analyses were conducted using Stata V.13.1.

\section{Ethical approval}

All participants provided electronic consent for the baseline assessments and the register linkage. The study protocol is available online (http://www.ukbiobank.ac.uk/).

\section{RESULTS}

Descriptive statistics are shown in table 1 (for descriptive statistics according to social isolation and loneliness status, please see online supplementary etables 1 and 2; for complete and imputed variable frequencies, please see online supplementary etable 3 ). Nine per cent of the individuals were socially isolated, $6 \%$ lonely, and 1\% isolated and lonely. From the socially isolated individuals, $16 \%$ were lonely, and from the individuals who were lonely, 23\% were socially isolated. Socially isolated and lonely individuals had higher prevalence of chronic diseases and current smoking. In addition, lonely individuals reported more depressive symptoms than non-lonely individuals. The mean follow-up was 7.1 years (range 5.4 to 10.0 years). Over the follow-up period, a total of 12428 participants died, 5731 had AMI and 3471 had stroke. Of the 5731 participants who had AMI, 900 died (16\%) during follow-up, and of the 3471 participants who had incident stroke, 844 died (24\%) over the follow-up.

The associations of social isolation with incident AMI and stroke are shown in figure 1 . In analyses adjusted for age, sex and ethnicity, social isolation was associated with higher risk 
Table 1 Descriptive characteristics of the study sample $(n=479054)$

\begin{tabular}{lc}
\hline & Mean (SD) or N (\%) \\
\hline Age (years) & $56.35(8.1)$ \\
\hline Sex & \\
\hline Women & $265702(55 \%)$ \\
\hline Men & $213352(45 \%)$ \\
\hline Ethnicity & \\
\hline Non-white & $25359(5 \%)$ \\
\hline White & $453695(95 \%)$ \\
\hline Deprivation Index & $-1.29(3.1)$ \\
\hline Education & \\
\hline No secondary education & $78454(17 \%)$ \\
\hline Secondary education & $236092(50 \%)$ \\
\hline University degree & $156466(33 \%)$ \\
\hline Household income & \\
\hline Less than $\mathrm{f} 31000$ & $89912(22 \%)$ \\
\hline f18000 to $f 29999$ & $103504(25 \%)$ \\
\hline$£ 30000$ to $f 51999$ & $107700(26 \%)$ \\
\hline f52 000 to $f 100000$ & $84590(21 \%)$ \\
\hline Greater than $\mathrm{f} 100000$ & $22557(6 \%)$ \\
\hline Chronic illness & $237287(51 \%)$ \\
\hline No & $227494(49 \%)$ \\
\hline Yes
\end{tabular}

Social isolation

$\begin{array}{lc}\text { No } & 427709(91 \%) \\ \text { Yes } & 42595(9 \%) \\ \text { Loneliness } & \\ \quad \text { No } & 428722(94 \%) \\ \text { Yes } & 28513(6 \%) \\ \text { Body mass index }\left(\mathrm{kg} / \mathrm{m}^{2}\right) & 27.35(4.75) \\ \text { Diastolic blood pressure }(\mathrm{mm} \mathrm{Hg}) & 82.3(10.12) \\ \text { Systolic blood pressure }(\mathrm{mm} \mathrm{Hg}) & 137.81(18.65) \\ \text { Handgrip strength }(\mathrm{kg}) & 30.55(11.01) \\ \text { Smoker } & \end{array}$

\begin{tabular}{lc}
\hline Smoker & $427738(90 \%)$ \\
\hline No & $49646(10 \%)$ \\
\hline Yes & \\
\hline Ex-smoker & $314466(66 \%)$ \\
\hline No & $162918(34 \%)$ \\
\hline Yes & $269812(56 \%)$ \\
\hline Alcohol consumption & $208893(44 \%)$ \\
\hline Twice or less per week & $3.59(2.33)$ \\
\hline At least three times per week & $1.87(1.95)$ \\
\hline Moderate physical activity* & $1.29(0.6)$ \\
\hline Vigorous physical activity* & $1.27(0.6)$ \\
\hline Depressed mood (range 1-4) & $1.31(0.6)$ \\
\hline Unenthusiasm/disinterest (range 1-4) & $1.68(0.81)$ \\
\hline Tenseness/restlessness (range 1-4) & \\
\hline Tiredness/lethargy (range 1-4) & \\
\hline Dee to missing dita covar & \\
\hline
\end{tabular}

Due to missing data in covariates, frequencies may not add up to the total number of participants.

*Number of days per week of physical activity lasting more than $10 \mathrm{~min}$.

of AMI (HR 1.43, 95\% CI 1.32 to $1.55, \mathrm{P}<0.001$ ). This association was attenuated by $14 \%$ after adjustment for biological factors, by $50 \%$ after adjustment for health behaviours, by $28 \%$ after adjustment for depressive symptoms, by $48 \%$ after adjustment for socioeconomic factors and by $16 \%$ after adjustment for chronic diseases. In the final model adjusted for all risk factors, the association was attenuated by $84 \%$ to 1.07 (95\%
CI 0.99 to 1.16 ) and did not remain statistically significant $(\mathrm{P}=0.109)$.

Social isolation was also associated with higher risk of incident stroke (HR 1.39, 95\% CI 1.25 to $1.54, \mathrm{P}<0.001)$ in the analyses adjusted for age, sex and ethnicity. The association attenuated by $14 \%$ after adjustment for biological factors, by $38 \%$ after adjustment for health behaviours, by $23 \%$ after adjustment for depressive symptoms, by $55 \%$ after adjustment for socioeconomic factors and by $15 \%$ after adjustment for chronic diseases. When adjusted for all risk factors, the association was attenuated by $83 \%$ to $1.06(95 \%$ CI 0.96 to 1.19$)$ and was not statistically significant $(\mathrm{P}=0.256)$.

The associations between loneliness with incident AMI and stroke are shown in figure 2. In analyses adjusted for age, sex and ethnicity, social isolation was associated with higher risk of AMI (HR 1.49, 95\% CI 1.36 to $1.64, \mathrm{P}<0.001$ ). This association decreased by $16 \%$ after adjustment for biological factors, by $35 \%$ after adjustment for health behaviours, by $62 \%$ after adjustment for depressive symptoms, by $39 \%$ after adjustment for socioeconomic factors and by $20 \%$ after adjustment for chronic disease. In the final model adjusted for all risk factors, the association did not remain statistically significant $(\mathrm{P}=0.235)$ and was attenuated by $87 \%$ to 1.06 (95\% CI 0.96 to 1.17$)$.

Loneliness was associated with higher risk of incident stroke (HR $1.36,95 \%$ CI 1.20 to $1.55, \mathrm{P}<0.001$ ) in the analyses adjusted for sex, age and ethnicity. The association attenuated by $16 \%$ after adjustment for biological factors, by $29 \%$ after adjustment for health behaviours, by $60 \%$ after adjustment for depressive symptoms, by $45 \%$ after adjustment for socioeconomic factors and by $21 \%$ after adjustment for chronic diseases. In the final model, adjusted for all risk factors, the association was attenuated by $89 \%$ to 1.05 (95\% CI 0.92 to $1.21)$ and did not remain statistically significant $(\mathrm{P}=0.577)$.

When loneliness, social isolation and the interaction between social isolation and loneliness were entered in the same model, social isolation and loneliness were associated with higher risk of AMI (social isolation: HR 1.36, 95\% CI 1.25 to $1.49, \mathrm{P}<0.001$; loneliness: HR $1.42,95 \% \mathrm{CI} 1.27$ to $1.59, \mathrm{P}<0.001$ ) and incident stroke (social isolation: HR 1.37, 95\% CI 1.22 to $1.54, \mathrm{P}<0.001$; loneliness: HR $1.35,95 \% \mathrm{CI}$ 1.17 to $1.56, \mathrm{P}<0.001$ ) in the analyses additionally adjusted for sex, age and ethnicity. The interaction terms between social isolation and loneliness were not statistically significant (all $\mathrm{P}>0.05$ ).

Figure 3 shows the associations between social isolation with mortality among participants who had incident AMI or stroke. Social isolation was associated with higher risk of mortality after AMI (HR 1.50, 95\% CI 1.25 to $1.79, \mathrm{P}<0.001$ ) in the analyses adjusted for age, sex and ethnicity. This association decreased by $13 \%$ after adjustment for biological factors, by $24 \%$ after adjustment for health behaviours, by $8 \%$ after adjustment for depressive symptoms, by $33 \%$ after adjustment for socioeconomic factors and by $9 \%$ after adjustment for chronic disease. In the final model adjusted for all risk factors, the association was attenuated by $50 \%$ to 1.25 (95\% CI 1.03 to 1.51 ), but remained statistically significant $(\mathrm{P}=0.023)$.

Similarly, in the analyses adjusted for age, sex and ethnicity, social isolation was associated with higher risk of mortality after stroke (HR $1.51,95 \%$ CI 1.25 to $1.83, \mathrm{P}<0.001$ ). This association decreased by $5 \%$ after adjustment for biological factors, by $24 \%$ after adjustment for health behaviours, by $7 \%$ after adjustment for depressive symptoms, by $26 \%$ after adjustment for socioeconomic factors and by $7 \%$ after adjustment for chronic disease. Finally, the association attenuated by $38 \%$ to 


\section{Social isolation}

AMI

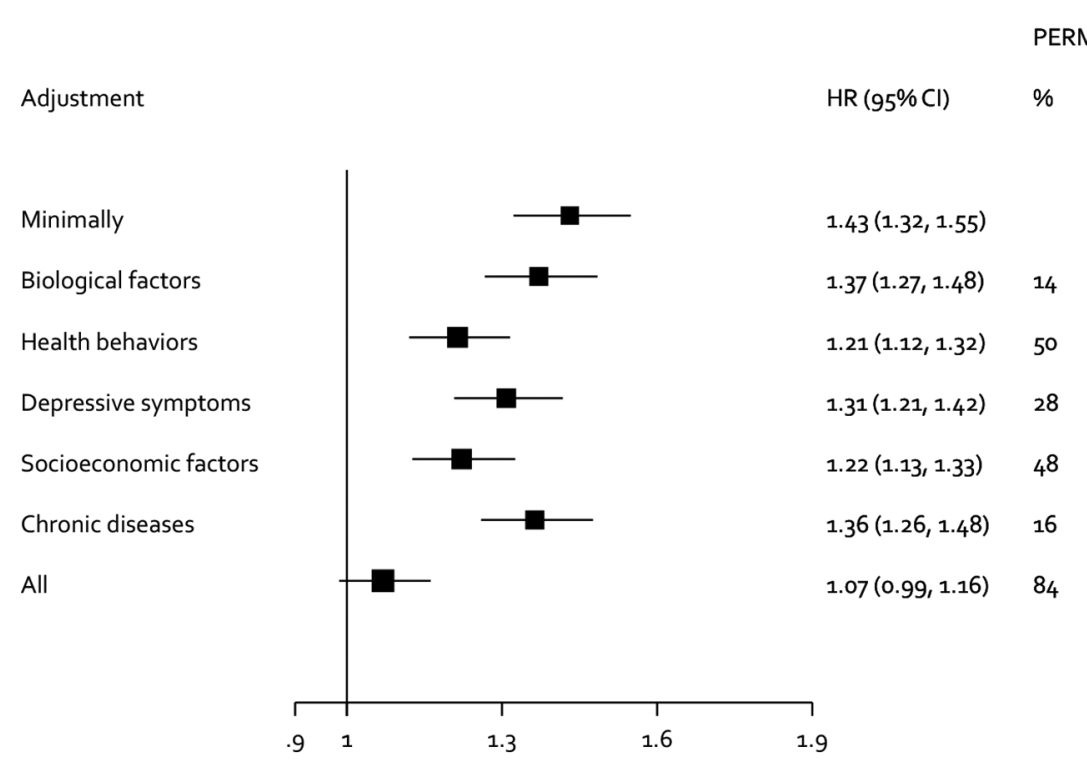

Stroke

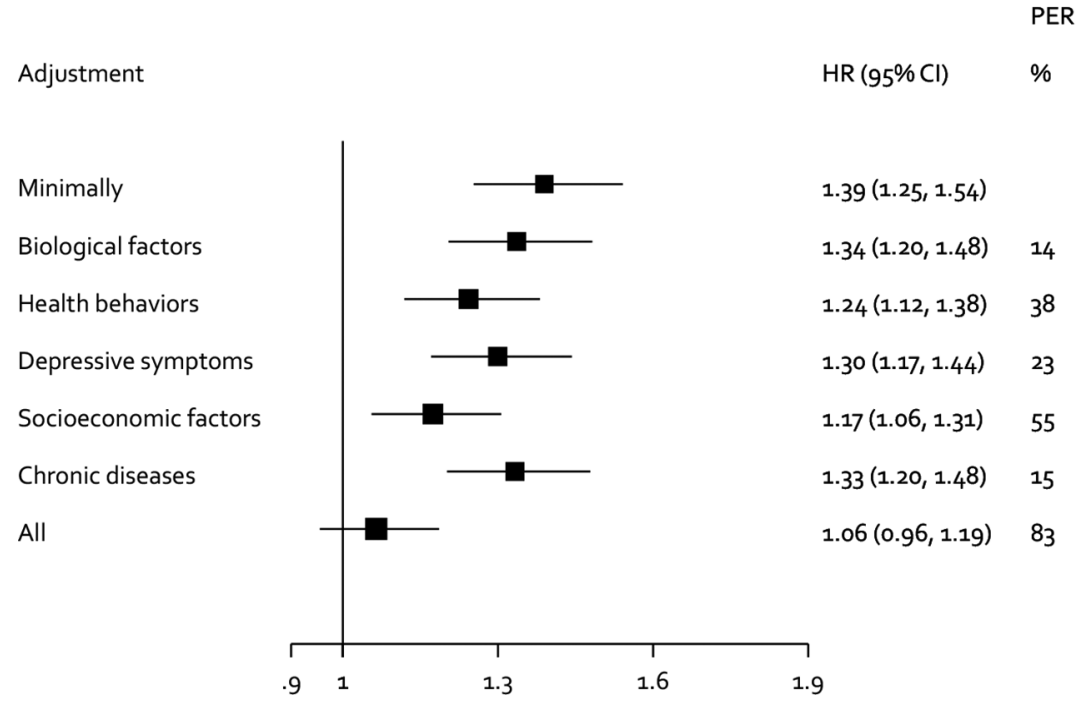

Figure 1 Proportions of the social isolation—AMI and stroke excess risk mediated by biological, behavioural, socioeconomic and health-related factors. AMI, acute myocardial infarction; PERM, percentage of excess risk mediated.

1.32 (95\% CI 1.08 to 1.61$)$, but remained statistically significant $(\mathrm{P}=0.007)$, in the final model adjusted for all risk factors. Loneliness, in turn, was not associated with mortality among participants who had incident AMI or stroke (online supplementary efigure 1).

\section{Sensitivity analyses}

We performed a number of sensitivity analyses to examine the robustness of the findings. First, we examined the associations between social isolation and loneliness with AMI and stroke across potential confounders, that is, three age groups, sex and ethnicity. The results were consistent across three age groups and two ethnic groups, but the associations of social isolation and loneliness with AMI were slightly stronger in women than men (online supplementary efigures 2-3). Similarly, the association between social isolation and stroke was slightly stronger in women (online supplementary efigure 3). Second, we performed complete case analyses where participants with missing values were excluded (322 818 participants had complete data on social isolation and all covariates; 315231 participants had complete data on loneliness and all covariates). The results from the complete case analyses were similar to those previously reported (online supplementary efigures 4-5). Last, we analysed the associations between a single item of loneliness ("Do you 


\section{Loneliness}

AMI

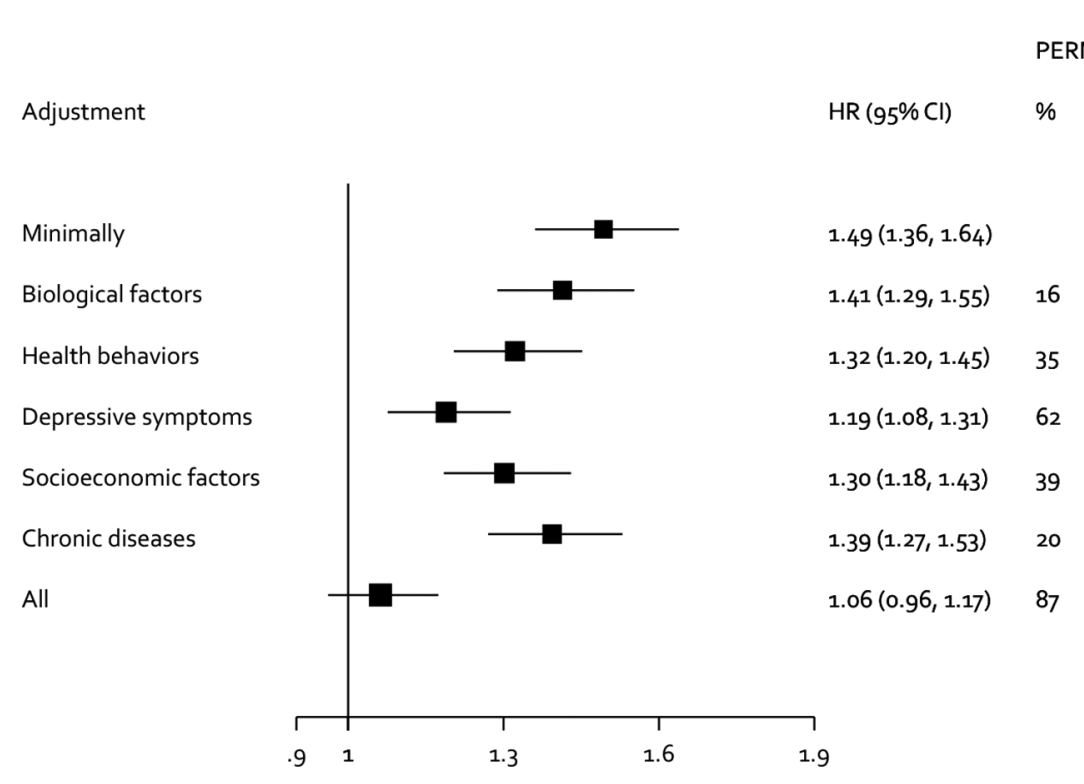

Stroke

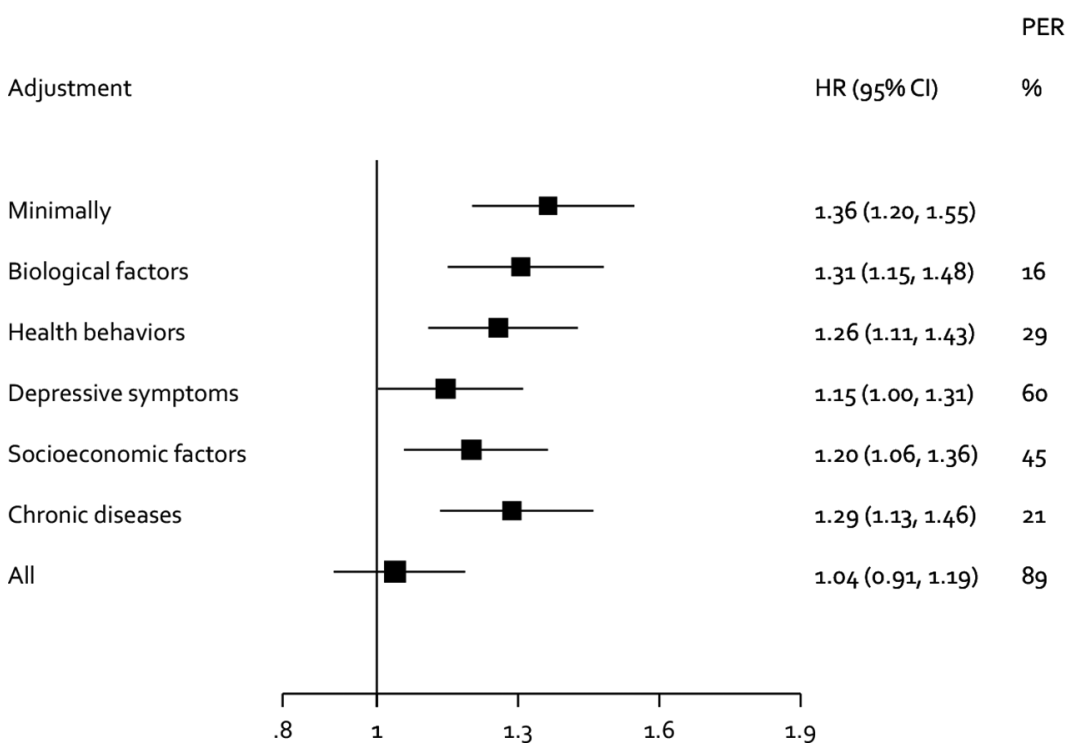

Figure 2 Proportions of the loneliness-AMI and stroke excess risk mediated by biological, behavioural, socioeconomic and health-related factors. AMI, acute myocardial infarction; PERM, percentage of excess risk mediated.

feel lonely?") with AMI and stroke. These associations were completely overlapping with the results from between loneliness with AMI and stroke (online supplementary efigure 6).

\section{DISCUSSION}

The main finding of this UK Biobank study of 479054 participants followed for over 7 years is that persons reporting social isolation and loneliness had 1.4-fold to 1.5-fold increased risk of incident AMI or stroke. However, approximately $85 \%$ of this excess risk was attributable to known risk factors such as obesity, smoking, low education and pre-existing chronic illness. In addition, social isolation, but not loneliness, was associated with 1.5-fold increased risk of mortality after the AMI or stroke event and although up to half of this excess risk was attributable to known risk factors, social isolation remained as an independent risk factor for mortality after the AMI and stroke event.

Our findings are in agreement with the previous studies where social isolation and loneliness have been associated with increased all-cause and cardiovascular disease mortality, ${ }^{3-7}$ and cardiovascular disease prognosis and incidence. ${ }^{24}$ Recent literature-based meta-analysis with 16 longitudinal studies showed that social isolation and loneliness are associated with $30 \%$ higher excess risk of stroke and cardiovascular heart disease after adjustment at least for age, gender and socioeconomic 


\section{Social isolation}

Mortality after AMI

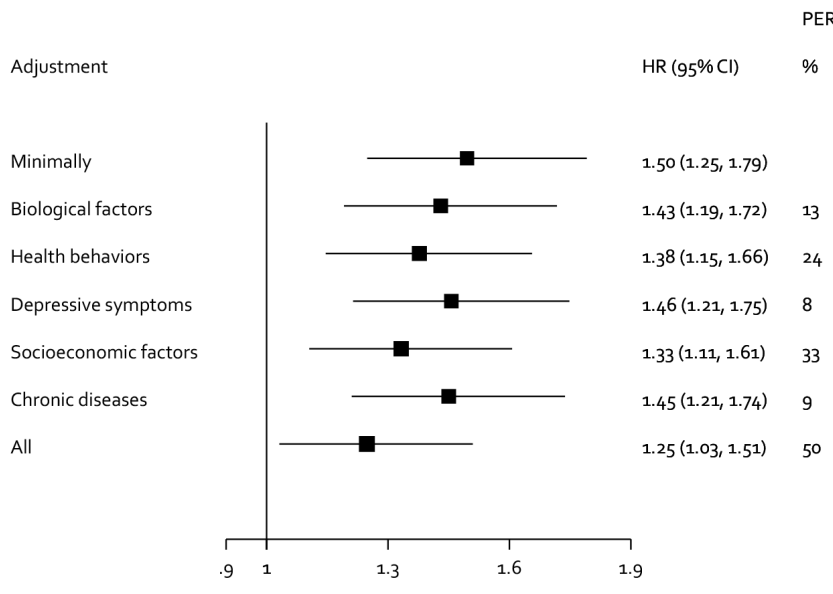

Mortality after stroke

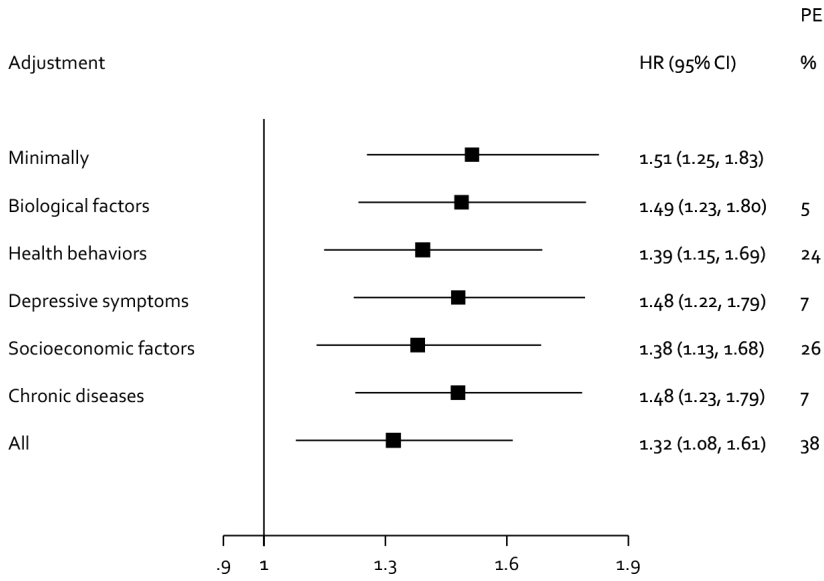

Figure 3 Proportions of the social isolation - mortality after AMI or stroke event excess risk mediated by biological, behavioural, socioeconomic and health-related factors. AMI, acute myocardial infarction; PERM, percentage of excess risk mediated.

status. ${ }^{8}$ Although these findings are of the same magnitude as ours before adjustment for risk factors and pre-existing chronic conditions, we were able to address the contribution of conventional risk factors to the association and we found that the associations were to a large extent attributable to these conventional risk factors. To the best of our knowledge, our study is the largest study on the topic. Differences between our findings and previous results could be related to study design or to selective publishing of positive results, which was suggested in the recent literature-based meta-analysis. ${ }^{8}$ In addition, it is possible that some of these adjustments lead to an underestimation of the true effect size, as social isolation and loneliness have been associated with many of these risk factors- such as depression ${ }^{25}$-and, thus, some of the mediators could also be confounders.

In our previous UK Biobank study with all-cause and cause-specific mortality as an outcome, we found similarly that the association between loneliness and cardiovascular mortality was fully explained by explanatory mechanisms, whereas the association between social isolation and all-cause mortality remained more independent. ${ }^{7}$ Thus, it seems that the association between social isolation and prognosis after a cardiovascular event is stronger than the association between loneliness and cardiovascular health. These findings indicate that social isolation, similarly to other risk factors such as depression, ${ }^{15}$ can be regarded as a risk factor for poor prognosis of individuals with cardiovascular disease.

Social isolation and loneliness can be seen as markers for many conventional risk factors-such as unhealthy lifestyles, poor mental health and socioeconomic adversity-and these risk factors also explain the association of social isolation and loneliness with cardiovascular morbidity. Thus, public health policies addressing conventional risk factors might also reduce the cardiovascular morbidity related to social isolation and loneliness. Further attention to social connections in public health prevention and intervention programmes could also potentially reduce the negative health outcomes of social isolation and loneliness. Importantly, guidance on how to address health risks associated with social isolation and loneliness could be added to the education of healthcare professionals, ${ }^{26}$ to promote prevention and treatment of cardiovascular disease in individuals with poor social connections.

\section{Strengths and limitations}

The UK Biobank is a large-scale prospective cohort study that provided a unique opportunity to examine our research question. Main outcomes (AMI, stroke and mortality) were acquired from health registers, and exposures (social isolation and loneliness) were self-reported. Social isolation was measured with three items and loneliness with two items. As it has been shown that multi-item assessment of social isolation has better predictive validity than single-item measures, ${ }^{4}$ multi-item assessment of social isolation and loneliness would have been a better option. Unfortunately, more items related to social isolation or loneliness were not available from the UK Biobank data. Although the response rate to UK Biobank was only $5.5 \%$, the participants are representative of the general population with respect to age, sex, ethnicity and deprivation within the recruitment age range. ${ }^{27}$ If the drop-out is non-random and related to social isolation or loneliness, this could bias the results leading either overestimates or underestimates of the studied associations. These issues, however, do not affect generalisability of our results as population prevalence and incidence rates were not the target of our study. Reverse causality-which previous studies have demonstrated ${ }^{28}$ - could bias our findings. However, participants with cardiovascular disease or stroke events before the study baseline were excluded from the analysis. As only the date of the first cardiovascular disease or stroke event is currently available from the UK Biobank data, we were not able to examine the association between social isolation and loneliness with recurrent cardiovascular disease stroke or events. This issue is likely to be important, as around one-fourth of strokes are recurrent, ${ }^{12}$ and social isolation before stroke has been shown to predict poorer outcomes after stroke. ${ }^{29}$ However, our results showed that social isolation is associated with increased risk of mortality after AMI or stroke event, indicating that social isolation is associated with poorer prognosis after AMI or stroke. Although we measured only social networks in a very simple way, studies using more complex measures have reported similar findings. ${ }^{2}$ Naturally, there is a possibility of residual confounding that cannot be completely ruled out in an observational study. UK Biobank included participants aged between 40 and 69, hence current findings may not be generalised beyond this age range.

\section{CONCLUSIONS}

Social isolation and loneliness are associated with increased risk of AMI and stroke. In addition, social isolation is related 
to elevated mortality after the incidence of AMI or stroke. However, although these associations are largely explained by other cardiovascular health risk factors and pre-existing chronic conditions, social isolation seems to remain as an independent risk factor for mortality after the AMI and stroke event.

Key messages

\section{What is already known on this subject?}

- Social isolation and loneliness have been associated with higher risk of cardiovascular disease and poorer prognosis, but it remains unclear whether these associations are independent of conventional risk factors.

\section{What might this study add?}

- In this population-based cohort study of over 470000 participants, most of the excess risk of cardiovascular disease and death after the cardiovascular event among isolated and lonely persons was explained by conventional risk factors.

\section{How might this impact on clinical practice?}

- Targeting conventional risk factors could reduce cardiovascular disease burden among isolated and lonely individuals.

Contributors $\mathrm{CH}$ and ME were responsible for the design of the study. $\mathrm{CH}$ conducted statistical analyses and wrote the first draft of the manuscript. All authors interpreted the data and critically revised the manuscript for important intellectual content. $\mathrm{CH}$ is the guarantor.

Funding This work was supported by the Academy of Finland (310591 to $\mathrm{CH}$, 311492 to MK, 258598 and 292824 to MV), NordForsk (MK) and Helsinki Institute of Life Science fellowship (MK).

Competing interests None declared.

Patient consent Obtained.

Ethics approval The UK Biobank study was approved by the NHS National Research Ethics Service (17 June 2011, Ref 11/NW/0382).

Provenance and peer review Not commissioned; externally peer reviewed.

Data sharing statement The present study has been conducted using the UK Biobank Resource (Application Number 14801) that is available to researchers (see http://www.ukbiobank.ac.uk)

(c) Article author(s) (or their employer(s) unless otherwise stated in the text of the article) 2018. All rights reserved. No commercial use is permitted unless otherwise expressly granted.

\section{REFERENCES}

1 Eng PM, Rimm EB, Fitzmaurice G, et al. Social ties and change in social ties in relation to subsequent total and cause-specific mortality and coronary heart disease incidence in men. Am J Epidemiol 2002;155:700-9.

2 Nagayoshi M, Everson-Rose SA, Iso H, et al. Social network, social support, and risk of incident stroke: Atherosclerosis Risk in Communities study. Stroke 2014;45:2868-73.

3 Steptoe A, Shankar A, Demakakos P, et al. Social isolation, loneliness, and all-cause mortality in older men and women. Proc Natl Acad Sci U S A 2013;110:5797-801.
4 Holt-Lunstad J, Smith TB, Baker M, et al. Loneliness and social isolation as risk factors for mortality: a meta-analytic review. Perspect Psychol Sci 2015;10:227-37.

5 Holt-Lunstad J, Smith TB, Layton JB. Social relationships and mortality risk: a metaanalytic review. PLOS Med 2010;7:e1000316.

6 Berkman LF, Syme SL. Social networks, host resistance, and mortality: a nine-year follow-up study of Alameda County residents. Am J Epidemiol 1979;109:186-204.

7 Elovainio M, Hakulinen C, Pulkki-Råback L, et al. Contribution of risk factors to excess mortality in isolated and lonely individuals: an analysis of data from the UK Biobank cohort study. Lancet Public Health 2017;2:e260-6.

8 Valtorta NK, Kanaan M, Gilbody S, et al. Loneliness and social isolation as risk factors for coronary heart disease and stroke: systematic review and meta-analysis of longitudinal observational studies. Heart 2016;102:1009-16.

9 Thoits PA. Mechanisms linking social ties and support to physical and mental health. J Health Soc Behav 2011:52:145-61.

10 Cacioppo JT, Cacioppo S, Capitanio JP, et al. The neuroendocrinology of social isolation. Annu Rev Psychol 2015;66:733-67.

11 Chen E, Miller GE. Socioeconomic status and health: mediating and moderating factors. Annu Rev Clin Psychol 2013;9:723-49.

12 Mozaffarian D, Benjamin EJ, Go AS, et al. Executive Summary: Heart Disease and Stroke Statistics - 2016 Update: a report from the American Heart Association. Circulation 2016:133:447-54.

13 Sarwar N, Gao P, Seshasai SR, et al. Diabetes mellitus, fasting blood glucose concentration, and risk of vascular disease: a collaborative meta-analysis of 102 prospective studies. Lancet 2010;375:2215-22.

14 Mons $\mathrm{U}$, Hahmann H, Brenner H. A reverse J-shaped association of leisure time physical activity with prognosis in patients with stable coronary heart disease: evidence from a large cohort with repeated measurements. Heart 2014;100:1043-9.

15 Lichtman JH, Froelicher ES, Blumenthal JA, et al. Depression as a risk factor for poor prognosis among patients with acute coronary syndrome: systematic review and recommendations: a scientific statement from the American Heart Association. Circulation 2014;129:1350-69.

16 Sudlow C, Gallacher J, Allen N, et al. UK biobank: an open access resource for identifying the causes of a wide range of complex diseases of middle and old age. PLoS Med 2015:12:e1001779-10.

17 Palmer LJ. UK Biobank: bank on it. Lancet 2007;369:1980-2

18 Townsend P, Phillimore P, Beattie A. Health and deprivation: inequality and the North. Kent, UK: Croom Helm, 1988

19 Hughes ME, Waite LJ, Hawkley LC, et al. A short scale for measuring loneliness in large surveys: results from two population-based studies. Res Aging 2004;26:655-72.

20 Spitzer RL, Kroenke K, Williams JB. Validation and utility of a self-report version of PRIME-MD: the PHQ primary care study. Primary Care Evaluation of Mental Disorders. Patient Health Questionnaire. JAMA 1999;282:1737-44.

21 Thiébaut AC, Bénichou J. Choice of time-scale in Cox's model analysis of epidemiologic cohort data: a simulation study. Stat Med 2004;23:3803-20.

22 Lin DY, Fleming TR, De Gruttola V. Estimating the proportion of treatment effect explained by a surrogate marker. Stat Med 1997;16:1515-27.

23 White IR, Royston P, Wood AM. Multiple imputation using chained equations: issues and guidance for practice. Stat Med 2011;30:377-99.

24 Steptoe A, Kivimäki M. Stress and cardiovascular disease: an update on current knowledge. Annu Rev Public Health 2013:34:337-54

25 Cacioppo JT, Hughes ME, Waite LJ, et al. Loneliness as a specific risk factor for depressive symptoms: cross-sectional and longitudinal analyses. Psychol Aging 2006:21:140-51.

26 Holt-Lunstad J, Smith TB. Loneliness and social isolation as risk factors for CVD: implications for evidence-based patient care and scientific inquiry. Heart 2016:102:987-9.

27 Collins R. What makes UK Biobank special? Lancet 2012:379:1173-4.

28 Hakulinen C, Pulkki-Råback L, Jokela M, et al. Structural and functional aspects of social support as predictors of mental and physical health trajectories: Whitehall II cohort study. J Epidemiol Community Health 2016;70:710-5

29 Boden-Albala B, Litwak E, Elkind MS, et al. Social isolation and outcomes post stroke. Neurology 2005;64:1888-92. 
Correction: Social isolation and loneliness as risk factors for myocardial infarction, stroke and mortality: UK Biobank cohort study of 479054 men and women

Hakulinen C, Pulkki-Råback L, Virtanen M, et al. Social isolation and loneliness as risk factors for myocardial infarction, stroke and mortality: UK Biobank cohort study of 479054 men and women. Heart 2018;104:1536-42. doi: 10.1136/heartjnl-2017-312663

In this article, the section entitled Procedures included the following text 'Loneliness was measured with two questions: "Do you often feel lonely?" (no=0, yes=1) and "How often are you able to confide in someone close to you?" ( $0=$ almost daily to once every few months; $1=$ never or almost never)'.

The latter query should have read ' $(0=$ almost daily to about once a month; $1=$ once every few months to never or almost never)'.

(c) Author(s) (or their employer(s)) 2019. No commercial re-use. See rights and permissions. Published by BMJ.

Heart 2019;105:e8. doi:10.1136/heartjnl-2017-312663corr1

A) Check for updates 